\title{
Surface Reflectance Modeling of Real Objects with Interreflections
}

\author{
Takashi Machidał, Naokazu Yokoyał, and Haruo Takemura $\nmid$ \\ $\dagger$ Cybermedia Center, Osaka University \\ $\ddagger$ Graduate School of Information Science, Nara Institute of Science and Technology \\ E-mail: machida@ime.cmc.osaka-u.ac.jp, yokoya@is.aist-nara.ac.jp, takemura@ime.cmc.osaka-u.ac.jp
}

\begin{abstract}
In mixed reality, especially in augmented virtuality which virtualizes real objects, it is important to estimate object surface reflectance properties to render the objects under arbitrary illumination conditions. Though several methods have been explored to estimate the surface reflectance properties, it is still difficult to estimate surface reflectance parameters faithfully for complex objects which have non-uniform surface reflectance properties and exhibit interreflections. This paper describes a new method for densely estimating non-uniform surface reflectance properties of real objects constructed of convex and concave surfaces with interreflections. We use registered range and surface color texture images obtained by a laser rangefinder. Experiments show the usefulness of the proposed method.
\end{abstract}

\section{Introduction}

In mixed reality (MR), it is needed that a virtualized object is seamlessly merged into the real world [1]. In order to represent a virtualized object photo-realistically, there are two approaches to the problem of virtualizing real objects. One is called an image based rendering (IBR) that has often been used to reproduce real objects in computer graphics (CG). Generally, IBR methods require a large number of real images to represent the virtualized object under arbitrary illumination conditions and arbitrary viewing directions. Mukaigawa et al. [15] have proposed a photometric IBR in which a virtualized object is represented with a few real images. However, in the case of using a limited number of images, a problem occurs; i.e., the appearance of object is not faithfully reproduced since some part of the object is interpolated linearly. The other method is called a polygon based rendering (PBR). This method reproduces the object shape and surface reflectance properties. If the object surface reflectance properties are estimated at once, the virtualized object can be rendered appropriately under virtualized illumination conditions estimated from real en- vironments $[5,16]$. A number of methods for estimating reflectance properties of an object surface have been developed $[2,8,9,10,11,13,17]$. In these methods, surface reflectance models with several parameters are employed, and shape and color information of the object are used to estimate the reflectance parameters.

Object surface reflection consists of diffuse and specular components. The diffuse reflection component is easily observed due to its nature of reflection in omni-direction. On the other hand, the specular reflection component is limitedly observed within a fixed range of angles with respect to a viewing position, light source and object surface normal vector. In some works $[2,8,13]$, it is assumed that an object has a uniform reflectance property over the entire surface. Reflectance parameters are estimated by using the standard least-squares method to fit a reflectance model to a given color image. Due to the assumption, such methods cannot be applied to objects which consist of several different materials and have non-uniform reflectance properties. On the other hand, to treat non-uniform surface objects, some works have used multiple images of an object under different lighting conditions and have estimated reflectance parameters by solving simultaneous equations $[9,10,11]$. However, such methods still have a problem such that results are not stable especially when the specular reflection component is very small.

Sato et al. [17] have developed a methodology to estimate non-uniform reflectance properties. They set up an object on a robot arm and measure the object with a CCD camera and a rangefinder from a large number of viewpoints by rotating the robot arm. In the method, reflectance parameters are stably acquired by decomposing the surface reflection into two components based on the singular value decomposition (SVD) technique. Although the method can be applied to objects with non-uniform reflectance properties, the shape of object should be limited. This is because it is difficult to observe the specular reflection component over the entire surface, since the lighting condition for a pose of the object against the camera cannot be changed in the method. 
Moreover, if the object has a complicated shape, interreflections may occur. Here, we take notice of the radiosity. The radiosity models interreflections in the real world. This method can represent interreflections based on the transmission of heat energy of light. Fournier et al. [7] have originally developed a method for estimating surface reflectance properties using the radiosity method in an indoor scene. This method assumes uniform reflectance on an object surface and determines only the diffuse reflectance parameter. There are some other attempts to estimate both diffuse and specular reflectance properties of a room $[3,12,20]$. They, however, also assume that all objects have the uniform reflectance properties in the region, therefore, their algorithm also cannot be applied to an object which has non-uniform surface reflectance properties.

In this paper, we propose a new method for densely estimating non-uniform reflectance properties of objects with interreflections. First, we select optimum light positions in order to decompose diffuse and specular components at each surface point. Then, we densely estimate the object surface reflectance parameters considering interreflections on the surface. The proposed reflectance modeling method is based on the radiosity and Torrance-Sparrow model. In experiments, the proposed method is qualitatively and quantitatively evaluated with reflectance parameter estimation and virtual relighting. The radiosity method can usually be applied to a closed environment. However, we experimentally verify that the radiosity can also be applied to an open environment in which the influence of the environment light is negligible.

\section{Surface reflectance modeling from range and color images}

Figure 1 illustrates a flow diagram of surface reflectance modeling. Our process consists of three parts: measurement of an object (A, C), selection of light source positions (B), and estimation of reflectance parameters (D, E).

1. Measurement of 3-D geometry and surface texture An object shape is acquired with a laser rangefinder in a dark room. Multiple surface texture images are also obtained under different light sources whose positions are selected in the process (B).

2. Selection of light source

According to the object shape and the position of the camera, multiple light positions are automatically selected among a number of possible positions of the light source to measure both diffuse and specular reflection components densely on the surface.

3. Estimation of reflectance parameters

Reflectance parameters are estimated by substituting

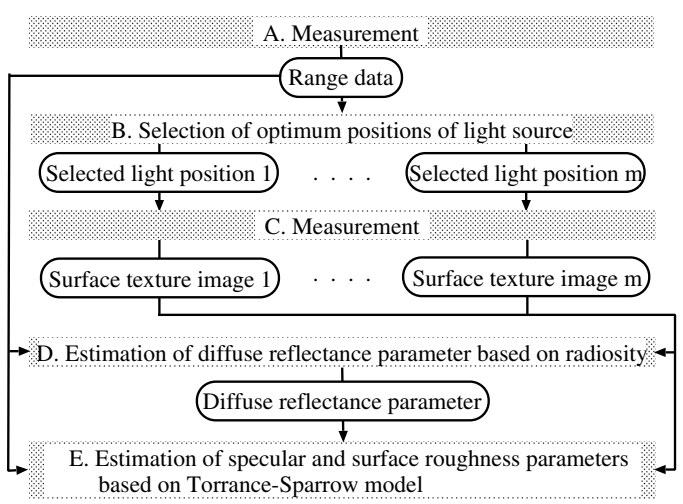

\section{Figure 1. Flow diagram of surface reflectance modeling.}

the object shape and textures under selected illumination conditions into the surface color reflection model. Surface interreflections are considered in this process.

\subsection{D Measurement of object}

We use a laser rangefinder (Cyberware 3030RGB) with known positions of point light sources and a camera for acquiring range and surface color images, which is located in a dark room as shown in Figure 2(a). This system can simultaneously obtain registered range and surface color texture images rotating the rangefinder and the camera around an object. Figure 2(b) shows the illustration viewed from the top of the device. A camera is located at $X 1$ and a texture image is acquired through mirrors which are located at $X 2$ and $X 3$. We thus assume that the camera is virtually located at $X 4$ and the camera looks toward the center of rotation.

Here, because a range image has some noises including quantization errors, we locally fit the range image to quadratic surfaces to acquire the normal vector correctly by using the Yokoya-Levine operator [19].

\subsection{Selection of positions of light sources}

Here we address the problem of determining light source positions for effectively observing diffuse and specular reflections on an object surface. In the present experimental setup, multiple positions of a light are determined among 60 possible positions prepared around the laser rangefinder and these are two-dimensionally arranged at the interval of $5 \mathrm{~cm}$ as shown in Figure 3. The positions of a camera and a light source are calibrated in advance. When optimum light positions are selected, a single light is attached at the selected positions in turn. Therefore, the calibration of brightness among multiple lights is not needed.

In this study, we employ the Torrance-Sparrow model [18] to represent surface reflections physically. The 


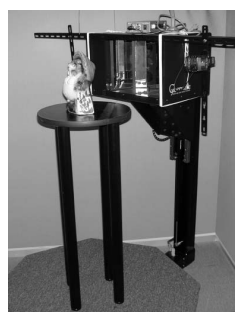

(a) Appearance of 3D-Digitizer

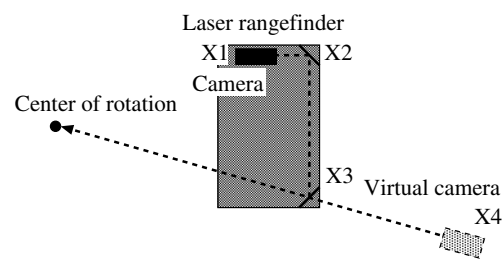

(b) Direction of camera
Figure 2. 3D-Digitizer.

Torrance-Sparrow model is given as:

$$
i=\frac{Y}{D^{2}}\left\{P_{d} \cos \theta_{d}+\frac{P_{s}}{\cos \theta_{v}} \exp \left(-\frac{\theta_{r}^{2}}{2 \sigma^{2}}\right)\right\},
$$

where $i$ represents an observed intensity, $D$ is an attenuation coefficient concerning the distance between a point light source and an object surface point, $Y$ represents the strength of a light source. $P_{d}, P_{s}$ and $\sigma$ are the diffuse reflectance, the specular reflectance and the surface roughness parameters, respectively. $\theta_{d}$ is an angle between a light source vector and a surface normal vector, $\theta_{v}$ is an angle between a viewing vector and a surface normal vector, and $\theta_{r}$ is an angle between a viewing vector and a reflection vector. Note that the reflection vector is a vector to which a light vector is mirrored against a normal vector. $\theta_{r}$ is used for judging whether the specular reflection occurs or not.

To densely estimate non-uniform reflectance parameters, it is required to observe each surface point under at least three different lighting conditions: One for determining one unknown parameter $P_{d}$ and others for acquiring two unknown parameters $P_{s}$ and $\sigma$. Therefore, the selection of light source position is repeated until almost all pixels in the range image satisfies three different lighting conditions. As a result of this process, a certain number of light positions, say $m$, are selected to densely observe both diffuse and specular reflection components.

Let $I_{p}$ be a color image which is to be obtained with a possible light position $p(p=1, \cdots, 60)$ and consists of $n$ pixels $\left(i_{p 1}, \cdots, i_{p n}\right)$, where $i_{p k}$ means a color intensity,

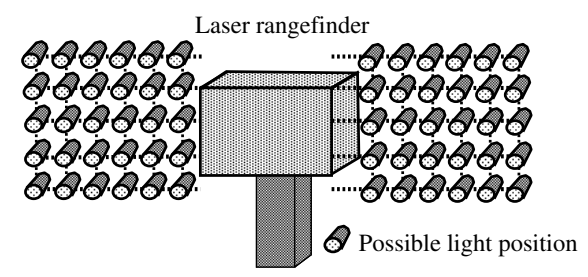

\section{Figure 3. Multiple possible light source posi- tions.}

$D_{p}$ be the number of pixels which include only the diffuse reflection component in $I_{p}$, and $S_{p}$ be the number of pixels which include the strong specular reflection in $I_{p}$.

First, the following conditions are examined for each pixel in the object surface texture under each light position $p,:$

- Measurability of light reflection,

- Measurability of only diffuse reflection,

- Measurability of strong specular reflection.

Second, the light positions $p$ and $q$ which satisfy $D_{p}=$ $\operatorname{Max}\left(D_{1}, \cdots, D_{60}\right)$ and $S_{q}=\operatorname{Max}\left(S_{1}, \cdots, S_{60}\right)$ are selected. In the next light source position selection, the position which satisfies the same condition is selected among the rest except for light source positions decided so far. Then, $m$ light positions are selected to densely estimate reflectance parameters. The selection of light positions is repeated until almost all pixels are observed once for only the diffuse reflection component and twice for the strong specular reflection component.

We introduce a threshold $t h$, i.e., the ratio of the measurability of both reflection components to stop the process of selecting light positions. With respect to determining the threshold $t h$, we judge the ratio of the measurability of the specular reflection with all possible positions. This ratio is a limit of measuring the specular reflection component. Using the threshold, we can terminate the light selection process in the case that the ratio of measurability of specular reflection component cannot increase more, even if the number of positions of light source is increased. In such a way, reflectance parameters can be efficiently estimated almost the whole object surface using a limited number of texture images. In the following, the three measurability conditions above are described in detail.

\subsubsection{Measurability of light reflection}

In order to measure the light reflection at a specific point of the object surface, the surface point must be observable from the camera position and the light must illuminate the surface point. Thus the positional relationship among the camera, the surface point and the light source must satisfy the following conditions.

$$
\left(\mathbf{V}_{k} \cdot \mathbf{N}_{k}\right)>\mathbf{0},\left(\mathbf{L}_{p k} \cdot \mathbf{N}_{k}\right)>\mathbf{0},
$$

where $\mathbf{V}_{k}, \mathbf{L}_{p k}$, and $\mathbf{N}_{k}$ are the viewing direction, the light source direction, and the surface normal at the $k$-th pixel, respectively. Note that the viewing direction $\mathbf{V}_{k}$ and the surface normal $\mathbf{N}_{k}$ are independent of the light source position $p$. Even when the above equations are both satisfied, there is a possibility that a shadow is casted on the pixel. In other words, a point on the object may be covered by a shadow casted by the light source $p$. Such a case can be judged by an existing technique[6]. 


\subsubsection{Measurability of diffuse reflection only}

When the $k$-th pixel exhibits only the diffuse reflection, the reflection vector $\mathbf{L}_{p k}^{\prime}$ satisfies the following equation.

$$
\theta_{r}=\cos ^{-1}\left(\mathbf{V}_{k} \cdot \mathbf{L}_{p k}^{\prime}\right)>\theta_{t h 1},
$$

where $\theta_{t h 1}$ is a threshold angle between $\mathbf{V}_{k}$ and $\mathbf{L}_{p k}^{\prime}$. Eq. (3) implies that only the diffuse reflection component is observed if $\theta_{r}$ is greater than $\theta_{t h 1}$ as illustrated in Figure 4. When this condition stands and the pixel is not in a shadow, the pixel is judged to have diffuse reflection only and is counted in $D_{p}$.

\subsubsection{Measurability of strong specular reflection}

When the $k$-th pixel exhibits the strong specular reflection, the reflection vector $\mathbf{L}_{p k}^{\prime}$ satisfies the following equation.

$$
\theta_{r}=\cos ^{-1}\left(\mathbf{V}_{k} \cdot \mathbf{L}_{p k}^{\prime}\right) \leq \theta_{t h 2},
$$

where $\theta_{t h 2}$ is a threshold angle between $\mathbf{V}_{k}$ and $\mathbf{L}_{p k}^{\prime}$. Eq. (4) means that both the diffuse and specular reflection components are observed if $\theta_{r}$ is smaller than $\theta_{t h 2}$ as illustrated in Figure 5. The above condition is based on the fact that the specular reflection is observed strongly in a limited range of viewing angle. When this condition stands and the pixel is not in a shadow, the pixel is judged to have strong specular reflection and is counted in $S_{p}$.

\subsection{Estimation of reflectance parameters}

This section is concentrated on the determination of reflectance parameters from registrated range and color texture images. A texture image is obtained with a selected light position as described in the previous section. Each pixel is classified into three types $T_{\text {diff }}, T_{\text {spec }}$ and $T_{\text {none }}$. $T_{\text {diff }}$ means a pixel containing only the diffuse reflection component and $T_{\text {spec }}$ means a pixel containing strong specular reflection component. $T_{\text {none }}$ means a pixel which is classified neither $T_{\text {diff }}$ nor $T_{\text {spec }}$ and is not used to estimate reflectance parameters.

\subsubsection{Estimation of diffuse reflectance parameter based on radiosity}

In the following, we describe a new method for densely estimating diffuse reflectance parameter of objects with interreflections. We assume that interreflections do not have an influence from the specular reflection component but only from the diffuse reflection component. Therefore, pixels categorized in $T_{\text {diff }}$ are used in the estimation of diffuse reflectance parameter. In this paper, in order to consider interreflections, we employ the radiosity equation given as:

$$
B_{u}=E_{u}+P_{d u} \sum_{v=1}^{\alpha} B_{v} F_{u v},
$$

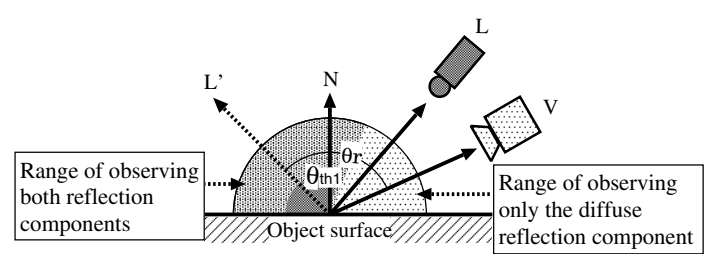

Figure 4. Observation of only the diffuse reflection.

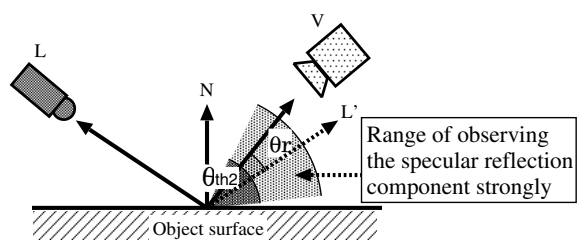

Figure 5. Observation of the specular reflection.

where $B_{u}$ and $B_{v}(1 \leq u, v \leq \alpha)$ represent the radiosity of $u$-th and $v$-th patches, respectively. Note that $\alpha$ is the number of object patches. $F_{u v}$ is a form factor between the patch $u$ and the patch $v . E_{u}$ represents an emission term which has an influence on the patch $u$ and actually $E_{u}$ means the light patch in the environment. Since the object is measured in a dark room in the present study, we can assume that the influence of environment light can be ignored. In other words, the form factor between each patch of the object and the wall of the dark room is not calculated. $P_{d u}$ represents the diffuse reflectance parameter on the patch $u$.

In the proposed method, the hemi cube method [4] can be used for calculating the form factor $F_{u v}$ because the object shape has already been measured by the laser rangefinder. Since the range and texture images are registered at each pixel, the radiosity $B_{u}$ of the patch $u$ is calculated based on the sum of the values of the pixels which correspond to the patch $u$. Then, the diffuse reflectance parameter $P_{d u}$ is determined as follows:

$$
P_{d u}=\frac{B_{u}-E_{u}}{\sum_{v=1}^{\alpha} B_{v} F_{u v}},
$$

where $E_{u}$ is 0 since there is no emission at the patch $u$ on the object. Finally, the diffuse reflectance parameter at each surface point is estimated by calculating the average among neighbouring patches which share the point.

Here, we describe the calculation of the radiosity $B_{u}$ of the patch $u$ in more detail. Each patch consists of four points and $B_{u}$ is represented as the sum of the color values of the pixels which correspond to these points. Let us suppose that the patch $u$ contains pixels $s$ and $t$ as shown in Figure 6. It should be noted that pixel values $e_{s}$ and $e_{t}$ of $s$ and $t$ in $T_{d i f f}$ are obtained with different light positions $p(s)$ and $p(t)$, respectively, because the light source attached with the rangefinder moves during measurement. In calculating the $B_{u}$, it is required to use the color value $\widehat{e_{t}}$ 


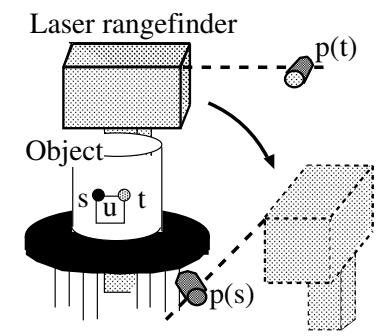

Figure 6. Calculation of radiosity.

of the pixel $t$ with the light position $p(s)$. From the Lambert's law, the color value $\widehat{e_{t}}$ can be obtained by:

$$
\widehat{\epsilon_{t}}=\frac{\mathbf{N}_{t} \cdot \mathbf{L}_{p(s) t}}{\mathbf{N}_{s} \cdot \mathbf{L}_{p(s) s}} e_{s}
$$

where $\mathbf{N}_{s}$ and $\mathbf{N}_{t}$ are normal vectors at pixels $s$ and $t$. $\mathbf{L}_{p(s) s}$ and $\mathbf{L}_{p(s) t}$ are light vectors with the light position $p(s)$ at pixels $s$ and $t$. Note that in the case of $\mathbf{N} \cdot \mathbf{L} \leq 0, \widehat{e_{t}}$ should be 0 to consider the measurability of light reflection.

\subsubsection{Estimation of specular reflectance and sur- face roughness parameters based on Torrance- Sparrow model}

The specular reflectance parameter $P_{s k}$ and the surface roughness parameter $\sigma_{k}$ at the $k$-th pixel which is categorized in $T_{\text {spec }}$ are estimated by solving the simultaneous equation of Eq. (1) with the values of the specular reflection component extracted from two images taken under two different illumination conditions and $P_{d k}$ estimated above. Note that $P_{d k}$ estimated above should be scaled to Eq. (1) before computing the specular and surface roughness parameters.

When strong specular reflection is observed less than twice, the specular reflectance and surface roughness parameters cannot be uniquely determined and thus are obtained by linear interpolation within a $3 \times 3$ window. If the window is small compared with the area, values of the reflectance parameters are interpolated linearly by scanning the texture image horizontally.

\section{Experiments}

In experiments, we first demonstrate results of estimating non-uniform surface reflectance properties as well as the effectiveness of the light position selection mechanism. We then examine the effect of considering interreflections in surface reflectance parameter estimation using objects with uniform and non-uniform surface properties. We fix some parameters as $\theta_{t h 1}=60^{\circ}, \theta_{t h 2}=20^{\circ}, t h=80 \%$ in all the experiments.

\subsection{Dense estimation of surface reflectance pa- rameters}

Figure 7(a) shows an object with non-uniform diffuse and specular reflections which is used in the first experiment. The proposed light selection method has determined 12 light source positions for the Object A. Estimated parameters for the Object $\mathrm{A}$ are given in Figure 8(a) (d), where parameter values are coded in color or intensity in the cylindrical coordinates. Figure 8(a) illustrates the diffuse reflectance parameter estimated over the object surface. Figure 8(b) does the specular reflectance parameter. This image clearly shows that the specular reflectance parameters of the doll's beak and leg are different from the rest. Actually, the beak and legs are highly reflective as can be seen in Figure 7(a). Figure 8(c) shows the surface roughness parameter with gray-scale where the largest value is coded as white. This image means that the smaller the value is, the smoother the object surface is. Figure 8(d) shows the ratio of pixels where specular reflectance and surface roughness parameters are computed. The black part means that both parameters are not directly estimated.

Figure 8(e) and (f) illustrate the measurability of both reflection components with respect to the number of light sources. Note that the horizontal axis is represented until 20, since these graphs does not change even if all 60 possible positions are used. In Figure 8(e), with respect to the diffuse reflectance parameter, when the number of selected light source positions is 5 , the ratio of the measurability of the diffuse reflection component is $100 \%$. In Figure 8(f), as for the specular reflectance and the surface roughness parameters, even when the object is measured with all the possible positions, the ratio of the measurability of the specular reflection component is $83.53 \%$. In our method, the ratio of measurability of the specular reflection component is $83.46 \%$ with automatically selected 12 light source positions. Since this figure is comparable to that with all the possible light source positions, the experiment shows that the proposed method is effective. The measurability of both reflection components and the number of selected light sources for estimating reflectance parameters of Objects A and $\mathrm{C}$ are shown in Figure 7(d). The number in the bracket means the number of selected positions of light source required to estimate the diffuse reflectance parameter. Note that it takes about 4 hours to calculate the form factor and estimate reflectance parameters of each object with a PC (CPU: Pentium4 3.06GHz, Memory: 1GB).

\subsection{Effect of considering interreflections}

We show that the proposed method can remove the influence of interreflections in a preliminary experiment. We separate the Object B into two regions I and II as shown 


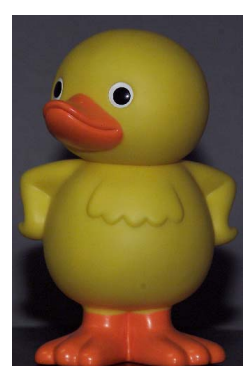

(a) Object $\mathrm{A}$

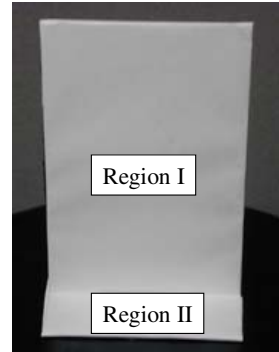

(b) Object B

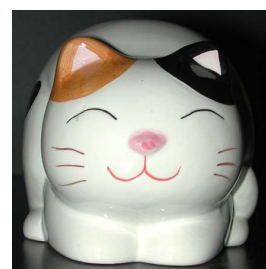

(c) Object C

\begin{tabular}{|c|c|c|}
\hline Object & A & C \\
\hline $\begin{array}{c}\text { Number of selected } \\
\text { light positions }\end{array}$ & $12(5)$ & $10(3)$ \\
\hline $\begin{array}{c}\text { Ratio of the measurability of } \\
\text { diffuse reflection component }\end{array}$ & $100.00 \%$ & $100.00 \%$ \\
\hline $\begin{array}{c}\text { Ratio of the measurability of } \\
\text { specular reflection component }\end{array}$ & $83.46 \%$ & $89.17 \%$ \\
\hline
\end{tabular}

(d) Measurability of both reflection components

Figure 7. Three objects used in experiments and the measurability of both reflection components.

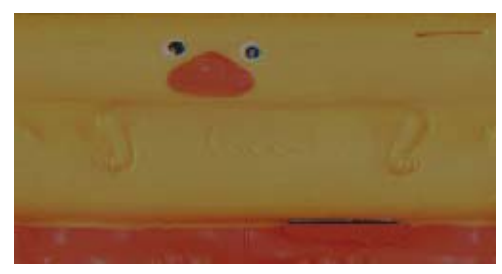

(a) Diffuse reflectance parameter $\left(P_{d}\right)$

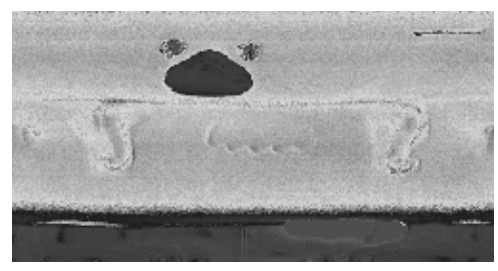

(c) Surface roughness parameter $(\sigma)$

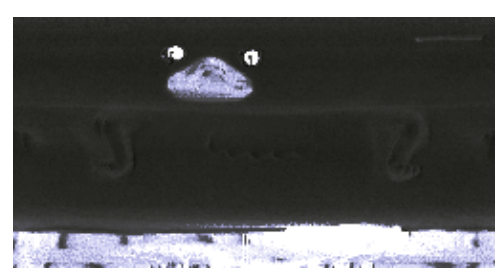

(b) Specular reflectance parameter

$\left(P_{s}\right)$

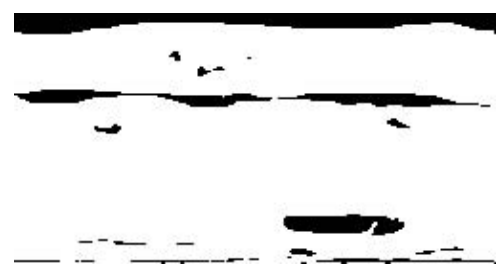

(d) Area of observing specular reflection

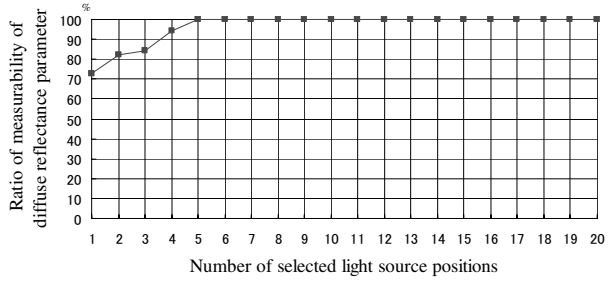

(e) Possibility of estimating the diffuse reflectance parameter

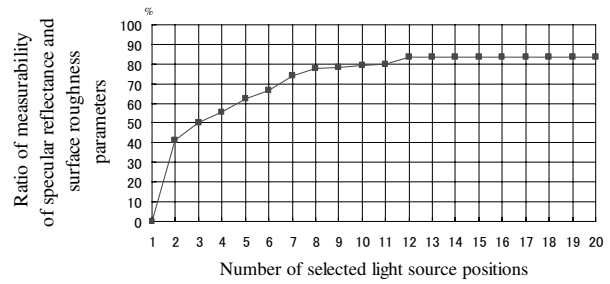

(f) Possibility of estimating the specular reflectance and the surface roughness parameter

Figure 8. Estimated reflectance parameters and measurability of both reflection components for Object A.

in Figure 7(b). We have conducted two setups. One is that the same white paper with a uniform diffuse reflectance surface is pasted up on both regions (setup1). The other is that the same glossy paper with a uniform reflectance surface is pasted up on both regions (setup2). In both setups, the Object $B$ is put on the table obliquely, so that the influence of interreflections can be observed. The proposed method is compared with our previous research [14] which does not consider interreflections in estimating reflectance parameters. It is expected that, if interreflections occur, the estimated value in the conventional method must be incorrect in that part. Results for diffuse (setup1) and specular (setup2) surfaces are shown in Figures 9 and 10, respectively. Each graph represents RGB channels of the diffuse reflectance parameter estimated by both methods. The horizontal axis means the position of the surface point in the vertical direction of the Object $B$ and the vertical axis means the average of diffuse reflectance parameters in the horizontal direction of the object. In Figure 9, it is observed that the diffuse reflectance parameters estimated by the conventional method are large around the boundary between the regions I and II. On the other hand, such an effect is eliminated in the proposed method. This clearly shows the effectiveness of the proposed method. However, the influence of interreflections still remains in the glossy surface as slightly observed in Figure 10, because interreflections due to the specular reflection also ocurr at the boundary between the regions I and II in the second setup.

The next experiment is conducted for showing the effect of considering interreflections in reflectance parameter estimation of non-uniform objects $\mathrm{A}$ and $\mathrm{C}$ shown in Figure 7. Figures 11(a) and (b) illustrate the sum of differences in RGB channels of the diffuse reflectance parameters between the conventional and proposed methods. These images are represented in the cylindrical coordinates with gray-scale where the maximum value is 255 . The difference 


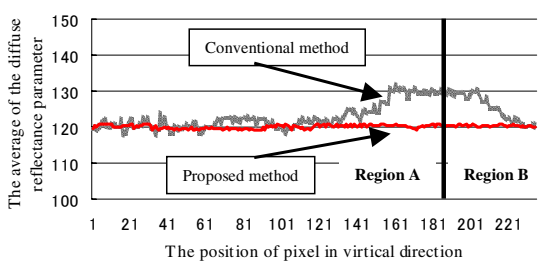

(a) R channel

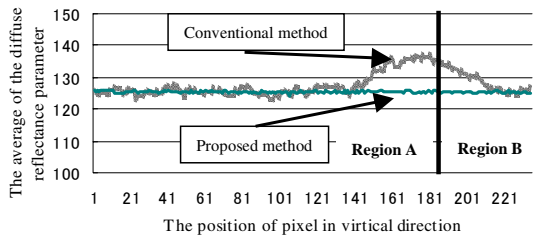

(b) G channel

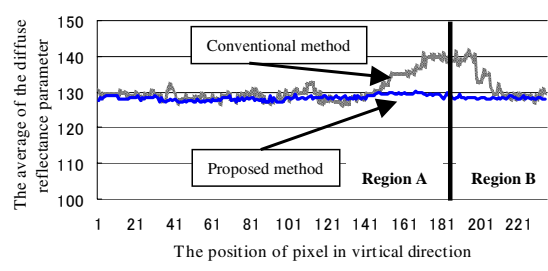

(c) B channel

Figure 9. A comparison with previous work for a uniform diffuse reflection surface in a preliminary experiment.

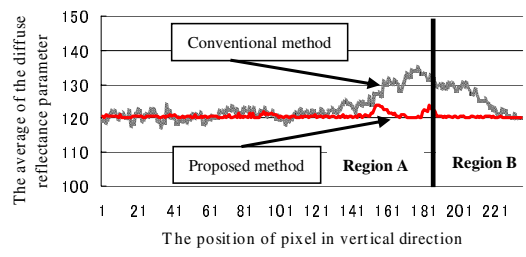

(a) R channel

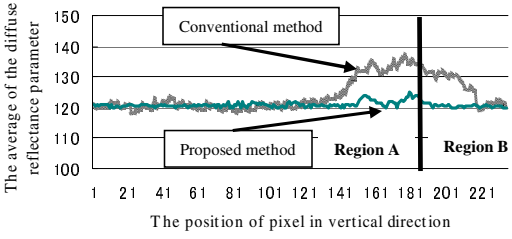

(b) G channel

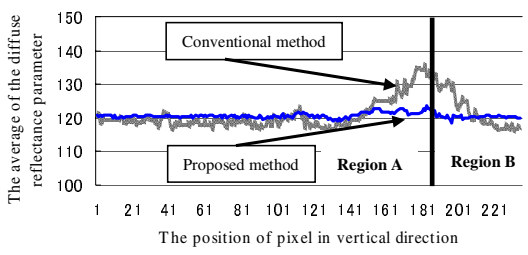

(c) B channel

Figure 10. A comparison with previous work for a uniform diffuse and specular reflection surface in a preliminary experiment.

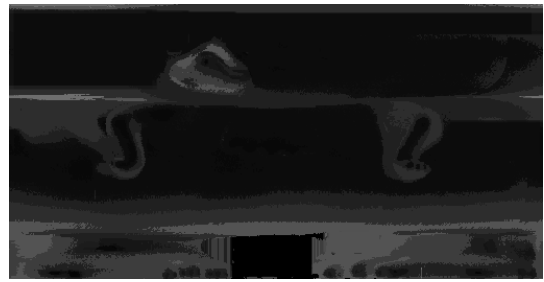

(a) Object A

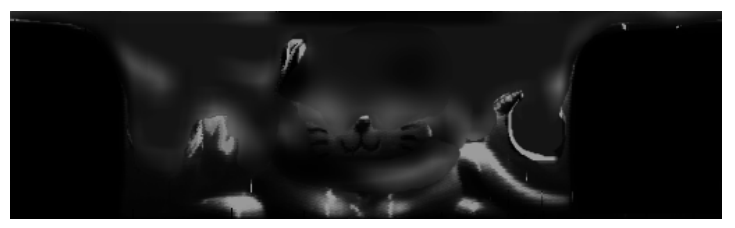

(b) Object C

Figure 11. Difference in estimated diffuse reflectance parameters between the proposed and conventional methods for Objects A and C.

is large in the parts where interreflections occur. These results show that the proposed method is effective for objects which have non-uniform surface reflectance properties and exhibit interreflections. There are some parts whose differences are larger than other parts. This is because the difference includes the influence of specular interreflections. In the proposed method, the diffuse reflectance parameter is calculated as the average of four patches as explained in Section 2.3.1. Therefore, the diffuse reflectance parameter which includes the influence of specular interreflections is also smoothed and that process causes a large difference compared with the conventional method.

\subsection{Rendering results with Torrance-Sparrow model under a virtual light source}

Finally, we demonstrate the virtual relighting of virtualized objects. Figure 12(a) and (b) show the rendering of virtualized objects $\mathrm{A}$ and $\mathrm{C}$ under a virtual lighting condition. A virtual light source is fixed at $(0.0,0.0,200.0)$ as the origin of the coordinate is at the center of the object. Each object rotates around the vertical axis of the object. Figures 12(a) and (b) contain three views such as right, front and left views, respectively. Each object is fairly rendered even at the part which contains interreflections. These results qualitatively show the effectiveness of the proposed surface reflectance modeling method.

\section{Conclusions}

In this paper, we have proposed a new surface reflectance modeling method which is based on densely estimating non-uniform reflectance properties for almost the whole object surface using the laser rangefinder for virtualizing real objects. In our approach, multiple light source positions around the laser rangefinder are automatically selected, so that diffuse and specular reflection components are observed densely. We have considered diffuse interreflections in estimating surface reflectance properties based on the radiosity. The experiments have proven that the proposed method is useful for estimating the reflectance parameters 


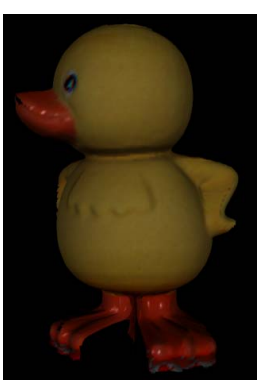

left view

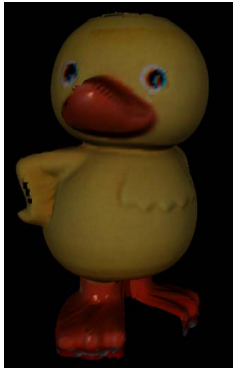

front view

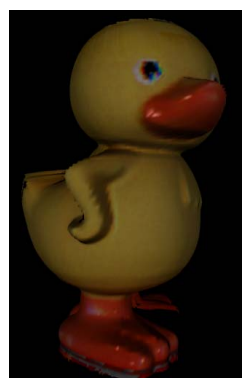

right view

(a) Rendering results of Object A

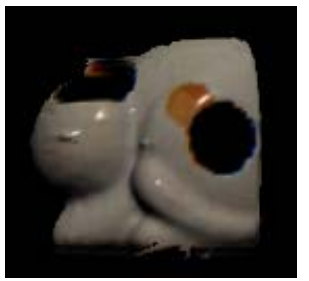

left view

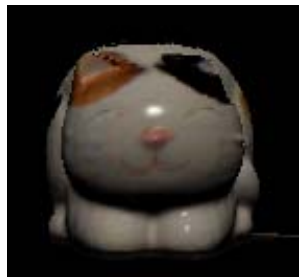

front view

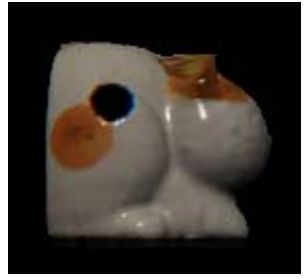

right view

(b) Rendering results of Object $\mathrm{C}$

of objects with diffuse interreflections which exhibit nonuniform surface reflectance.

In the future, we will extend the method for considering specular interreflections, which is suggested in experiments in Section 3.2. We will also investigate a method for automatically estimating illumination conditions in real environment which is required for merging virtualized objects into real images for mixed reality applications.

\section{Acknowledgments}

This work was supported in past by Grant-in-Aid for Scientific Research and also by NAIST-IS 21st Century COE Program from the Ministry of Education, Culture, Sports, Science and Technology.

\section{References}

[1] R. T. Azuma. A survey of augmented reality. Presence, 6(4):355-385, 1997.

[2] R. Baribeau, M. Rioux, and G. Godin. Color reflectance modeling using a polychromatic laser sensor. IEEE Trans. on Pattern Anal. Mach. Intell., 14(2):263-269, 1992.

[3] S. Boivin and A. Gagalowicz. Image-based rendering of diffuse, specular and glossy surfaces from a single image. Proc. SIGGRAPH 2001, pages 107-116, August 2001.

[4] M. F. Cohen and D. P. Greenberg. A radiosity solution for complex environments. Proc. SIGGRAPH 1985, pages 3140, 1985.

[5] P. Debevec. Rendering synthetic objects into real scenes: Bridging traditional and image-based graphics with global illumination and high dynamic range photography. Proc. SIGGRAPH' '98, pages 189-198, 1998.

[6] J. Foley, A. van Dam, S. Feiner, and J. Hughes. Computer Graphics Principles and Practice. Addison-Wesley Publishing Company, 2nd edition, 1993.

[7] A. Fournier, A. S. Gunawan, and C. Romanzin. Interactive common illumination for computer augmented reality. Proc. Graphics Interface 1993, pages 254-262, May 1993.

[8] K. Ikeuchi and K. Sato. Determining reflectance properties of an object using range and brightness images. IEEE Trans. on Pattern Anal. Mach. Intell., 13(11):1139-1153, 1991.
[9] G. Kay and T. Caelli. Inverting an illumination model from range and intensity maps. CVGIP: Image Understanding, 59:183-201, 1994.

[10] S. Lin and S. W. Lee. Estimation of diffuse and specular appearance. Proc. 7th Int. Conf. on Computer Vision, 2:855$860,1999$.

[11] S. Lin and S. W. Lee. A representation of specular appearance. Proc. 7th Int. Conf. on Computer Vision, 2:849-854, 1999.

[12] C. Loscos, G. Drettakis, and L. Robert. Interactive virtual relighting of real scenes. IEEE Trans. Visualization and Computer Graphics, 6(4):289-305, December 2000.

[13] J. Lu and J. Little. Reflectance function estimation and shape recovery from image sequence of a rotating object. Proc. 5th Int. Conf. on Computer Vision, pages 80-86, June 1995.

[14] T. Machida, H. Takemura, and N. Yokoya. Dense estimation of surface reflectance properties for merging virtualized objects into real images. Proc. 5th Asian Conf. on Computer Vision, pages 688-693, January 2002.

[15] Y. Mukaigawa, H. Miyaki, S. Mihashi, and T. Shakunaga. Photometric image-based rendering for image generation in arbitrary illumination. Proc. 8th Int. Conf. on Computer Vision, pages 643-649, July 2001.

[16] I. Sato, Y. Sato, and K. Ikeuchi. Acquiring a radiance distribution to superimpose virtual objects onto a real scene. IEEE Trans. on Visualization and Computer Graphics, 5(1):1-12, January 1999.

[17] Y. Sato, M. D. Wheeler, and K. Ikeuchi. Object shape and reflectance modeling from observation. Proc. SIGGRAPH '97, pages 379-387, 1997.

[18] K. E. Torrance and E. M. Sparrow. Theory for off-specular reflection from roughened surfaces. Journal of Optical Society of America, 57(9):1105-1114, 1967.

[19] N. Yokoya and M. D. Levine. Range image segmentation based on differential geometry: A hybrid approach. IEEE Trans. Pattern Anal. Mach. Intell., 11(6):643-649, June 1989.

[20] Y. Yu, P. E. Debevec, J. Malik, and T. Hawkins. Inverse global illumination: Recovering reflectance models of real scenes from photographs. Proc. SIGGRAPH '99, pages 215-227, August 1999. 